\title{
Application of an Aerothermal Model for Effusion Cooling Systems and Finite Rate Chemistry in Aero-Engine Combustors
}

\author{
T. Aumeier ${ }^{1}$ and T. Behrendt ${ }^{2}$ \\ ${ }^{1}$ Institute of Propulsion Technology, German Aerospace Center, \\ Linder Höhe, 51147 Köln, Germany, thomas.aumeier@dlr.de \\ ${ }^{2}$ Institute of Propulsion Technology, German Aerospace Center, \\ Linder Höhe, 51147 Köln, Germany, thomas.behrendt@dlr.de
}

\begin{abstract}
Effusion cooling is an advanced cooling concept for modern aeroengine combustors and offers a very stable convective coolant film along the wall combined with heat removal inside each hole. In order to find the best positions of the cooling holes, CFD calculations with conjugate heat transfer can be done to predict wall temperature distributions. An extensive CFD simulation with local grid refinement is required to evaluate near wall phenomena. A combustor with complex effusion hole pattern leads to large computational meshes for both the fluid and the solid increasing the computational costs. A helpful approach to speed up calculations is to replace the cooling holes by a set of new in- and outflow boundary conditions for virtual effusion holes. Computational costs and time for the design process can be saved. In the paper the cooling concept of a combustor liner with 368 virtual cooling holes is shown and the influence of the combustion near the wall is investigated. The combustion model used allows to account for kinetic effects in the near wall region.
\end{abstract}

\section{Introduction}

The fuel efficiency of an aero engine can be improved by increasing the overall temperature and pressure level leading to a higher thermodynamic efficiency. With conventional combustor technology, which can be found in almost any present gas turbine aero engine, this approach also increases the emissions of nitric oxides (NOx). The NOx production rates are highest in near stoichiometric regions, see e.g. Lefebre (1999) [1]. These regions are created when air jets are mixed into fuel rich combustor flow and are an inherent feature of the air staged combustion in conventional combustors. The fuel rich mixture injected by the burners is transformed into a fuel lean mixture. As the emission limits for NOx and also for carbon monoxide (CO) and unburned hydrocarbons (UHC) are becoming more and more restrictive, combustor development has to handle conflicting design targets (emissions versus fuel efficiency). The most promising approach to solve this design conflict is the concept of lean combustion. Here the air fuel mixture injected by the burners is already lean and in conjunction with partially pre-mixing the existence of near stoichiometric regions can be reduced significantly. Peak temperatures and thus NOx production rates are reduced substantially allowing a further increase of the overall temperature level to improve the fuel efficiency. In comparison to conventional combustors a complete redistribution of the air flow into the combustor is required which also affects the amount of air available for cooling the combustor walls leading to a sparse wall cooling film. Effusion cooling concepts are suitable as here the cooling film is refreshed continuously. The interaction between the highly turbulent, swirling and reacting burner flow field with the wall cooling film is the key to understand lean combustion in aero engine combustors under all operating conditions. Depending on the operating conditions the reacting fuel air mixture is prone to quenching due to local mixing effects with the cooling air and kinetic effects may cause increased CO emissions. Several approaches to simplify numerical calculations for effusion 
cooling concepts exist and an overview including a short description can be found in the work of Voigt et al. (2012) [2] and Facchini et al. (2014) [3]. Most of the models found in literature replace the cooling channels through the wall by the use of point or cell sources in the wall adjacent cells. The holes themselves are not represented in the geometrical setup and thus need not be meshed. The main objective is to reduce the costs of development and computing power while the degree of accuracy is expected to be reduced respectively. These models also operate with sources in the solid if conjugate heat transfer is considered and heat is exchanged with the solid. In [3] the implementation of a local pressure drop dependent formulation is presented which allows the automatic calculation of the mass flow rate through each modelled hole. While the mass flow rate could be specified as a fixed value it could be divided homogenously to all cooling holes in the work of [2]. In both cases heat transfer due to conduction in the solid could not be neglected and thus heat convection inside the cooling hole must be modelled additionally. Furthermore the results of an investigation of the influence of the grid size have been presented. In the work of Rida et al. (2012) [4] the authors reported an alternative method to the addition of source terms, hence represented by imprinted effusion modelling which consists in defining cold and hot sides of the effusion orifice with outlet and inlet boundary conditions but at adiabatic conditions only. The mass flow rate is calculated locally starting from the pressure drop and a correlation-based calculation of a discharge coefficient $c_{D}$. The main goal of our work is the usage of the boundary method compared to [4] with a forced mass flow assumption determined by the pressure drop over the hole in a detailed simulation. The boundary pattern of each virtual hole is mapped as good as possible to obtain the real hole cross section. Both the area and the shape of the modelled hole should match the real hole for the inlet and outlet ortifices. Of course the algorithm is limitded to the underlying mesh topology, the finer the mesh the better the approximation. Heat conduction inside the wall was included and heat convection in the virtual cooling holes was modelled as a heat sink for the solid as well as a heat source for the cooling air. This heat transfer model is coupled with a kinetically limited combustion model to enable investigations of the quenching impact on the $\mathrm{CO}$ oxidation at a later stage.

\section{The Effusion Hole Model}

\subsection{Basic Idea}

The core of the effusion hole model is to seperate the effusion cooling holes from the geometrical shape. Instead respective boundary patches are defined, that simulate the incoming and outgoing coolant flow of the modelled effusion hole which is shown in Fig. 1. Point A in Fig. 1 represents the inflow from the coolant channel into the virtual hole where point B represents the outflow out of the virtual hole into the hot flow domain.

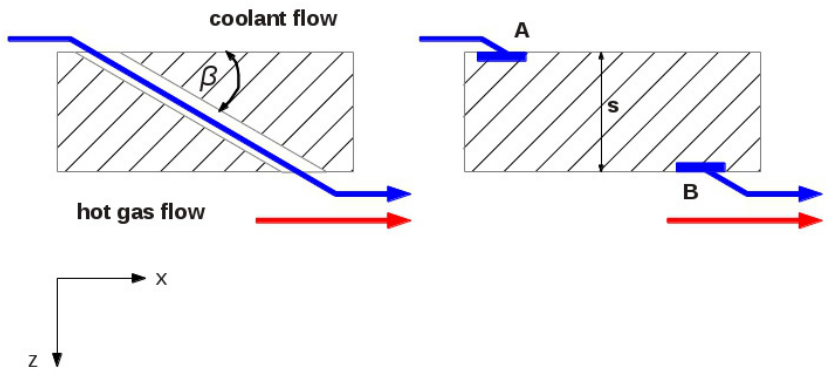

Figure 1: Basic idea of the effusion model
In our specific case the combustor wall has no holes left. Thus, the flow as well as the convective heat transfer inside the tube have to be modelled. For the flow an one dimensional momentum conservation equation based on a simple streamline theory can be used [3], [4]. The velocity at the hole exit is then calculated from pressure drop over the holes as follows 


$$
u=c_{D} \sqrt{\frac{2 \Delta p}{\rho}}
$$

Considering wall friction a $c_{D}$ correlation depending on the Reynolds number [5] is used. In fact, this velocity should represent the outflow velocity at the exit of the modelled effusion hole into the combustor. In practice the flow is much more complex and depending on the flow regime, a specific velocity profile at the cooling hole exit is present which is not considered in the presented model. The effusion holes are disposed to the coolant surface with a specific angle $\beta$. Thus, the velocity components must be adapted. In addition to velocity, temperature as well as turbulence parameters have to be defined at the new inflow boundary patches which represents the exit of the virtual hole. The temperature can simply set to the temperature of the coolant flow but if heat convection inside the coolant holes is considered a new temperature sould be calculated as described in section 2.3.

\subsection{Definition of New Boundaries}

Because of the design process the location of each virtual hole on the coolant side is given and indicates the geometrical center of the hole. The position of the new inflow boundary patch (point B in Fig. 1) can easily be calculated if the inclination angle is known. To set new boundary conditions both at point A and B surface elements are modified automatically from "wall" to "outflow" and "inflow" boundary, respectively. The main advantage of this approach is to reduced time for geometry and mesh generation. In contrary to use a source term formulation like in [2] or [3], no additional changes in the boundary conditions have to be applied. For numerical stability more than one surface element should be defined. At real conditions an inclined hole forms an elliptical cross section. Knowing point A and thus point B one also knows one surface element at each side.

An advanced searching algorithm is applied to find neighbouring surface elements as long as it allows to represent the real surface areas of the outgoing and incoming cross section as shown in Fig. 2. Overlapping the real cross section with the modelled section gives an impression about the differences between the real and the virtual configuration. The modelled one is almost equal to the real surface area and the position of the boundary inflow also represent the elliptic shape of the cross section. The finer the underlying grid the better the real surface area can be reproduced see Schroeder (2015) [6].

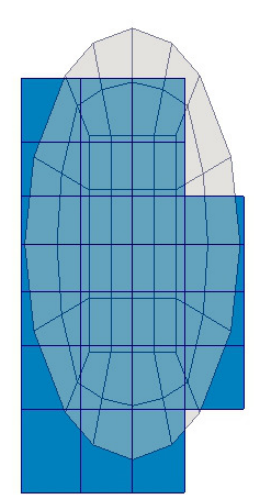

Figure 2: Real surface cross section vs. boundary face definitions

The influence of the mesh resolution on the flow field is investigated at a generic configuration consisting of a parallel coolant and a hot channel flow which is show in Fig. 3 . Effusion holes are used for cooling the surface on the hot gas side which are $0.6 \mathrm{~mm}$ in diameter and have an inclination angle of 30 degrees. In the generic configuration the coolant holes have been integrated into the computational domain. The advanced algorithm has been used to determine the input and outflow boundary patches for the effusion hole model. Several runs for different mesh resolutions have been performed [6]. The temperature distribution in the hot 
channal along a 1D line at $1 \mathrm{~mm}$ above the coolant hole is plottet in Fig, 3a for a fine and in Fig. $\mathrm{Bb}$ for a coarse mesh resolution. The results are in a good agreement to the generic reference configuration improving with mesh refinement.

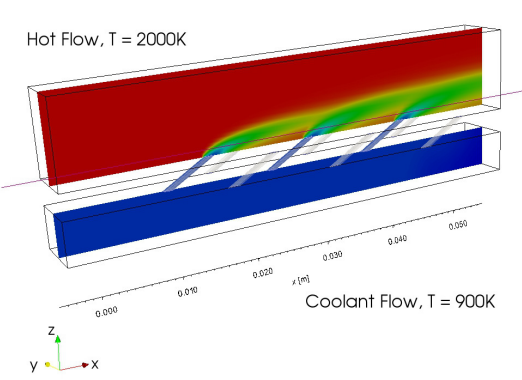

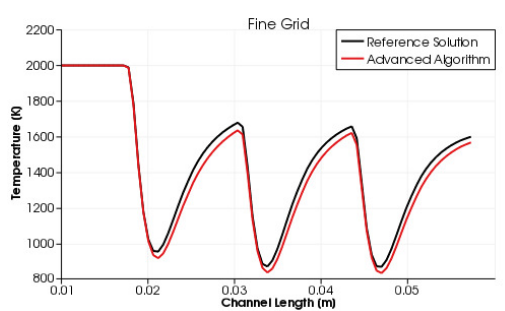

(a) Fine grid

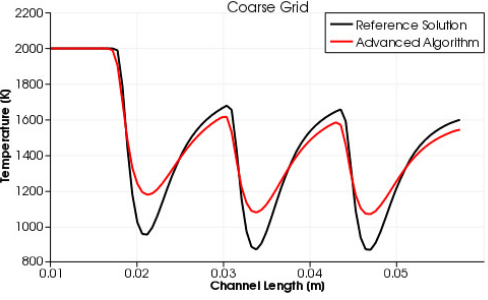

(b) Coarse grid

Figure 3: Temperature distribution of a generic configuration

\subsection{Heat Transfer to the Solid}

There is also convective heat transfer within the hole that leads to an increased outlet temperature of the coolant jet at the entrance to the hot gas flow domain. This temperature depends on the local wall temperatures which has to be calculated in an iterative procedure with the fluid solver. The temperature at the exit of the hole is calculated during the simulation as

$$
T_{\text {outlet }, \text { hole }}=T+\frac{\dot{Q}_{e f f}}{\dot{m}_{f l} c_{p}}
$$

$T_{\text {outlet, hole }}$ is the new inlet boundary condition for the solver, $T$ the coolant temperature when entering the hole, and $\dot{Q}_{e f f}$ the heat flow due to convective heat transfer from the wall to the fluid. Heat is transferred from the wall to the fluid and corresponds to a heat sink inside the solid wall with the same magnitude of $\dot{Q}_{e f f}$ but negative. In the presented case heat conduction in the wall is taken into account. A FEM solver is used and a solid-fluid coupling with conjugated heat transfer is realized in combination with the used CFD solver. The solid domain is meshed by tetrahedrons only, where the holes are omitted as well. The heat sink in the solid is then defined as

$$
\dot{Q}_{\text {solid }}=-\alpha_{\text {eff }} \cdot A_{\text {eff }} \cdot\left(T_{\text {solid }}-T_{\text {outlet }, \text { hole }}\right)
$$

where $\alpha_{e f f}$ is the average heat transfer coefficient in the hole, $A_{\text {eff }}$ the surface of the effusion hole and $T_{\text {solid }}$ the average wall temperature. Because the solid is dissolved by a computational grid, Eqn. (2) and Eqn. (3) can be discretized along the hole inside the wall as shown in Fig. 4.

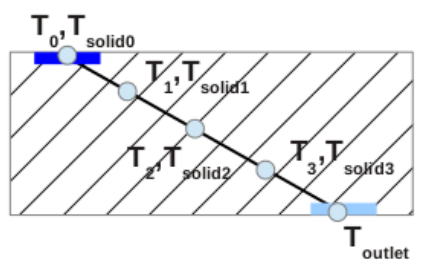

Figure 4: Discretization of the wall for conductive heat transfer
For each section of the hole (from index 0 to index 1 for example) a new temperature is calculated and used as input value for the following. Still the average heat transfer coefficient is used but the wall surface $A$ is adapted to the section. At the end of an iteration process the outlet temperature of the coolant $T_{\text {outlet, hole }}$ is calculated. The value of $\alpha_{e f f}$ is calculated as 


$$
\alpha_{e f f}=\frac{N u \cdot \lambda_{f l}}{d}
$$

while the value of $N u$ is taken from a correlation of the Nusselt number of turbulent pipe flows by Hausen [7] with

$$
N u=0.037\left(\operatorname{Re}^{0.75}-180\right) \operatorname{Pr}^{0.42}\left[1+\left(\frac{d}{l}\right)^{0.67}\right]
$$

The Prandtl number is set to $\operatorname{Pr}=0.7$. The Reynolds number is calculated using the velocity from Eqn. (1) and the hole diameter of $d=0.6 \mathrm{~mm}$. $\lambda_{f l}$ represents the thermal conductivity of the fluid. $l$ is the length of the hole with

$$
l=\frac{d}{s} \cdot \sin \beta
$$

where $s$ represents the wall thickness. The location of the sampling points for the calculation is done by searching over all solid cell elements in only one preprocessing step. These cells are marked and can be assigned after each iteration.

\section{Combustor Configuration}

A combined experimental and numerical approach was used to characterize the interaction between burner flow field and cooling film. The measurements were conducted in a single sector combustor with a rectangular cross section, which is operated at elevated pressures up to 5 bar; for a detailed description of the experimental approach see Lange et al. [8]. The work presented in this contribution is focused on the numerical approach. The investigated configuration consists of a burner and the combustor with cooled walls. The burner in Fig. $5 \mathrm{~b}$ is built of an annular nozzle with a total of 20 equally distributed radial swirler vanes. The fuel is injected by radial jets which consist of 40 feeding holes at the inner side in the center of the mixing region. In this way we intend to achieve a homogeneous premixed fuel-air distribution. The combustor has a quadratic cross section of $102 \mathrm{~mm} \times 102 \mathrm{~mm}$ with a total length of about $380 \mathrm{~mm}$. Only the front part is shown in Fig. 5b. On one side of the combustor wall a cooled wall element with 368 effusion holes is inserted. Because of optical access additional cooling air is needed for transparet wall elements. Combustion air is preheated and enters the combustor with a total mass flow of $80 \mathrm{~g} / \mathrm{s}$. For the fuel natural gas is used which is substituted by methane in the numerical simulation. The cooling air has a preheated temperature as well and a mass flow of $22.8 \mathrm{~g} / \mathrm{s}$ leading to a relative pressure drop across the cooled wall of $3.4 \%$. The effusion hole pattern is displayed in Fig. 5a. The wall element consists of 35 rows of effusion holes, 11 rows with 18 holes per row and 10 rows with 17 holes per row, respectively. The spacing can also be seen in Fig. 5a. The wall thickness of the plate is $1.5 \mathrm{~mm}$ and the diameter of the hole is 0.6 $\mathrm{mm}$. The wall element consists of Inconel 718.

\section{The Numerical Tools and Setup}

\subsection{The SATURNE and SYRTHES OpenSource Packages}

The open source CFD code CODE SATURNE (CS) 2.0.1 was used [9]. CS relies on a finite volume discretization and allows the use of various mesh types. But for numerical stability as well as a minimum amount of grid size only cube cells were created. The SALOME 7.4.0 integration platform was used to design the burner and the combustor respectively and to build 


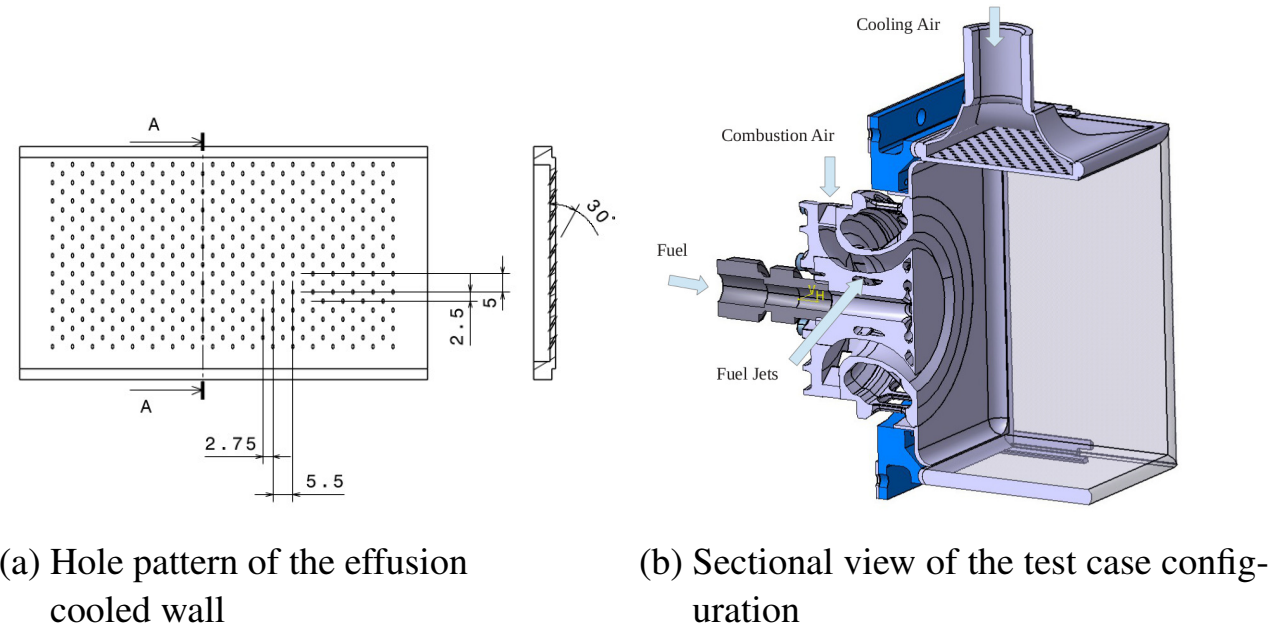

Figure 5: Geometrical case description

the computational mesh. Since the geometry of the test case is very complex, several parts were meshed independently from each other and joined with the help of joining function features of the preprocessor. Especially where the effusion holes hit both the combustor and the cooling channels a high resolution in contrary to the inner side of the combustor is needed but the joining leads to reduction of the grid resolution. Nevertheless a grid with a size of more than 1.6 million cells was created. In combination with the solid and thermal code SYRTHES [10] conjugate heat transfer problems are solved. SYRTHES is a thermal solver that is coupled with the fluid mechanics code CS. Heat transfer is essentially made by conduction in the solid, convection from the fluid to the solid and radiation. In the presented study only conduction in the solid is considered, which leads to the following equation for the solid part

$$
\rho c_{p} \frac{\partial T}{\partial t}=-\operatorname{div} \vec{q}+\Phi
$$

where $\rho$ and $c_{p}$ are the density and the heat capacity of the material considered. $\vec{q}$ represents the heat flux, and $\Phi$ a volumetric source term, which is calculated by the effusion hole model using Eqn. (3). $\dot{Q}_{\text {solid }}$ has to be transformed into $\mathrm{W} / \mathrm{m}^{3}$ to satisfy Eqn. (7).

\subsection{The Combustion Model}

For the numerical simulation of finite rate chemistry a turbulent combustion model (Partially Stirred Reactor Model - PaSR) was added to the solver that allows the calculation of complex chemical mechanism with a number of various species see Golovitschev et al. 1999 [11]. Some more detailed descriptions can be found in [12]. The model is not implemented by the basic solver. Hence, new scalar transport equations for the chemical species must be introduced where species sources due to turbulent combustion reaction rates are calculated. To guarantee mass conservation, the mass fraction of species $n$ is calculated by summing up the mass fraction of the $(n-1)$ species and subtract the result from unity. In our case $N_{2}$ is used as the species $n$ to minimize numerical error because it has the overall largest mass fraction. In addition, the enthalpy equation must be solved together with flow and species equations in order to ensure that the computed thermodynamic state is consistent with the ideal gas assumption. With a kinetically controlled combustion model the influence of the cooling air on the combustion process in the near wall reagions in addition with the heat transfer to the wall can be studied. 


\subsection{Numerical Setup}

The computational domain consists both of a fluid part involving the burner and combustor as well as a solid part involving the coolant wall element. In case of the fully resolved simulation the effusion hole geometry with 368 effusion holes is used in addition for the fluid part. The same configuration is considered by eliminating the effusion holes and using the effusion hole model instead. Due to simplification of the design process the same meshes for burner and combustor where used. The fluid domain is meshed with a total of about 1.65 million cube cells for the reference case. When the holes are omitted a mesh size of about 1.5 million cells remains. The mesh of the solid must be adjusted separately. Because tetrahedrons are used for the solid the mesh exceeds more then 0.92 million cells for the reference case but only 0.3 million for the case without effusion holes. Periodic boundary conditions for the burner, combustor and the solid wall element are assumed. Reynolds Averaged Navier-Stokes simulation has been performed with CS using an adapted $k-\epsilon$ turbulence model. Boundary conditions at the inlets are set as described in section 3 and summarized in Tab. 1. For the fuel methane is used. After each iteration a coupling step to SYRTHES was achieved to account for the conjugate heat transfer. For the solid the material properties are defind using the temperature dependend formulations for conductivity $\lambda$ and heat capacity $c_{p}$ of Inconel 718. The fluid pressure-velocity coupling is achieved through a segregated algorithm in which momentum components and pressure equation are solved in sequence so that the continuity equation is satisfied. In addition to pressure and velocity coupling, turbulence, species and enthalpy equations are solved. From fluid to the solid local temperatures and heat transfer coefficients closed to the wall are transferred while from the solid to the fluid the local wall temperatures are exchanged. A chemical mechanism consisting of 84 reactions and 21 species is used [13], where 20 species are transported by the solver. For the turbulent combustion source term modelling the PaSR model [11] is used. A characteristic chemical time scale for each reaction as well as a local turbulent mixing time is required for the model. The chemical time scale can be calculated using the kinetics given by the reaction mechanism. A good approximation of the mixing time is delivered by the Taylor scale and can be expressed as $\tau_{m i x}=c_{P a S R} k / \varepsilon$ [12] with $c_{P a S R}$ set to 0.005 .

Table 1: Boundary Conditions for the CFD

\begin{tabular}{|c|c|c|c|}
\hline & $\mathrm{T}[\mathrm{K}]$ & $\dot{m}[\mathrm{~kg} / \mathrm{s}]$ & $\mathrm{Tu}[\%]$ \\
\hline \hline Air $_{\text {burner }}$ & 450 & 80.0 & 10 \\
\hline Air $_{\text {cooling }}$ & 450 & 22.8 & 2 \\
\hline Fuel $_{\text {CH4 }}$ & 285 & 4.5 & 2 \\
\hline
\end{tabular}

\section{Results}

A complex simulation has been carried out, where the complete turbulent flowfield both in the burner and the combustor has been calculated. In addition a kinetically controlled combustion model has been used for heat release and species concentration prediction. Finally the effusion cooling system has been represented by 368 effusion cooling holes and a conjugate heat transfer problem with a metallic wall element was solved. All underlying physical processes have their particular advantages which should not be discussed in detail in this paper. The main focus is laid on the near wall region where cooling air enters the combustor. The same calculation on the same numerical grid but without effusion holes is done using the effusion model described 
above. In Fig. 6 the gas temperature distribution for the simulation with the real effusion holes (reference solution) in the wall is shown in a slice at $x=0 \mathrm{~mm}$. The flame is anchored at the burner exit and stabilized by an inner recirculation. Maximum calculated temperatures are about $2180 \mathrm{~K}$ even near the effusion cooled wall segment. Results from Lange et al. (2012) [8] are comparable to the calculated velocity distributions.

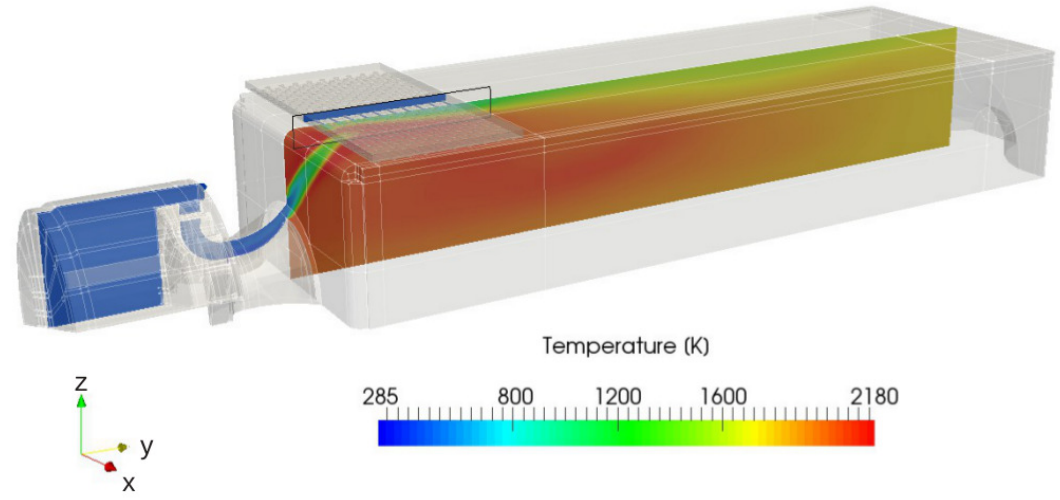

Figure 6: Temperature distribution of the reference solution in a slice at $x=0$

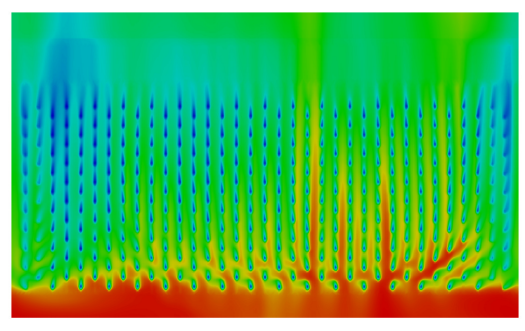

(a) Reference

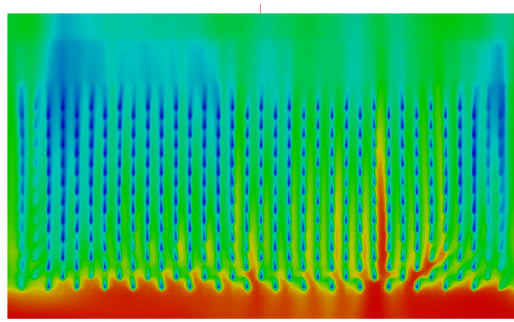

(b) Modelled

Temperature (K)

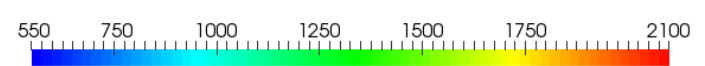

Figure 7: Gas temperature distribution parallel to cooled wall surface

Slices with temperature and velocity distribution at a distance of $0.5 \mathrm{~mm}$ parallel to the cooled wall are plotted in Fig. 7 and Fig. 8. As one can recognize the results for the reference and modelled simulation are in a good agreement. The flowfield is not symmetric and a maximum in the temperature distribution can be observed on the right hand side of the cooled wall element. Hot gas streaks are formed. The same results at $x=0 \mathrm{~mm}$ close to the wall in an band of about $6 \mathrm{~mm}$ are presented in Fig. 9 where also no significant differences between reference and modelled simulation may be observed at first view. The results are located within the black frame in Fig.6. The temperature distribution of the reference solution is given in Fig.9a whereas Fig. $9 \mathrm{~b}$ shows the calculation with the effusion hole model. The results are always scaled to the reference solution. In comparison to the reference case a slightly thicker cooling film may be observed in case of the modelled simulation in this region of the combustor. The black contour lines mark regions of specific temperature ranges. It can be seen that cooling air penetrates deeper into the combustor when using the effusion hole model but is still in good agreement to the reference case. The differences might be due to the simple calculation of the new inflow boundary condition using Eqn.1. On the other hand the virtual new inflow patch differs from 


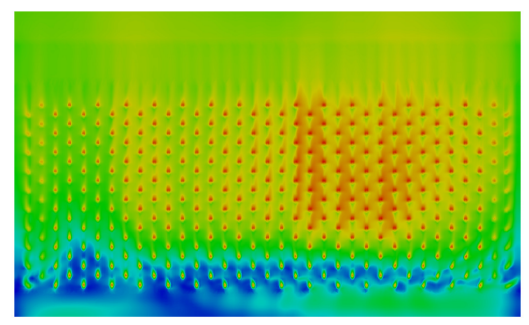

(a) Reference

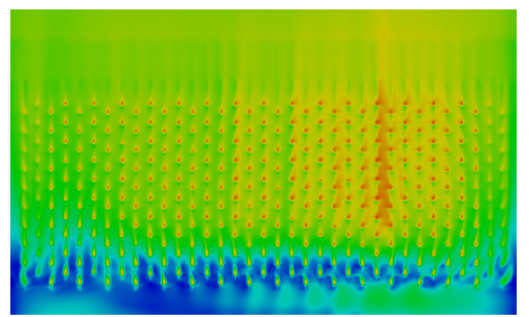

(b) Modelled

Velocity $(\mathrm{m} / \mathrm{s})$

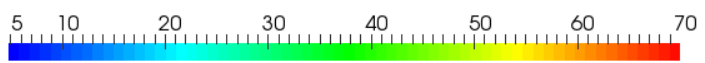

Figure 8: Velocity distribiution parallel to cooled wall surface

the real hole cross section. Furthermore instead of turbulent flow profiles simple block profiles have been used both for temperature and velocity at the new inflow patches. Also velocities are plotted in the same region and are compared with each other. Black contour lines mark regions of specific velocity ranges. Again the calculated velocity magnitude provides a similar agreement between reference and modelled simulation but is also nearby the reference solution. A better interpretation of the results can be done using line plots at a distance of dist $=0.5 \mathrm{~mm}$ from the cooled wall element. Fig 11 shows the results of the reference solution in comparison of two effusion model simulations: 1) conjugated heat transfer in the virtual holes is disabled in the calculation, i.e. assuming adiabatic conditions to the wall inside the hole and 2) conjugated heat transfer in the virtual holes is enabled in the calculation.

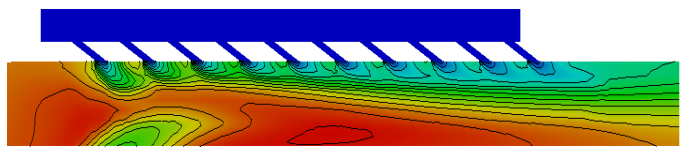

(a) Reference

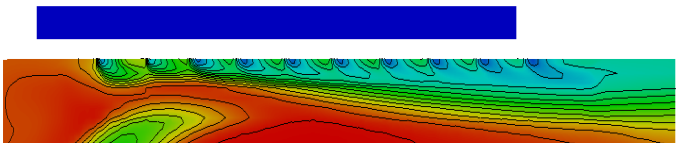

(b) Modelled

Temperature in $(K)$

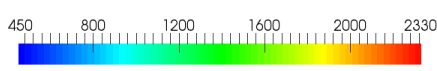

Figure 9: Temperature distribution

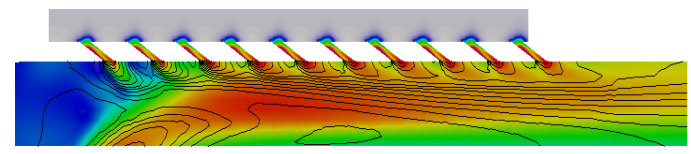

(a) Reference

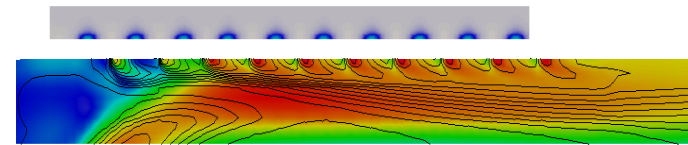

(b) Modelled

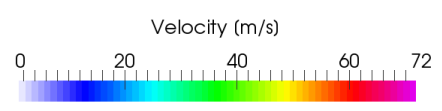

Figure 10: Velocity distribution

In the second case the new inflow temperature is heated due to convective heat transfer from the wall to the cooling air. In Fig. 11a velocity profiles and in Fig $11 \mathrm{~b}$ temperature profiles are shown. Close to the combustor head differences are larger than in the region where cooling air enters the combustor. Almost no deviations in the velocity profiles can be observed for the modelel simulation but tends to be better for the case if convective heat transfer in the virtual hole is taken into account. Also the temperature plot of the modelled simulation with heat convection in the hole is in better agreement with the reference solution compared to the adiabatic formulation. The cooling air temperature increases within the holes up to $80 \mathrm{~K}$. 


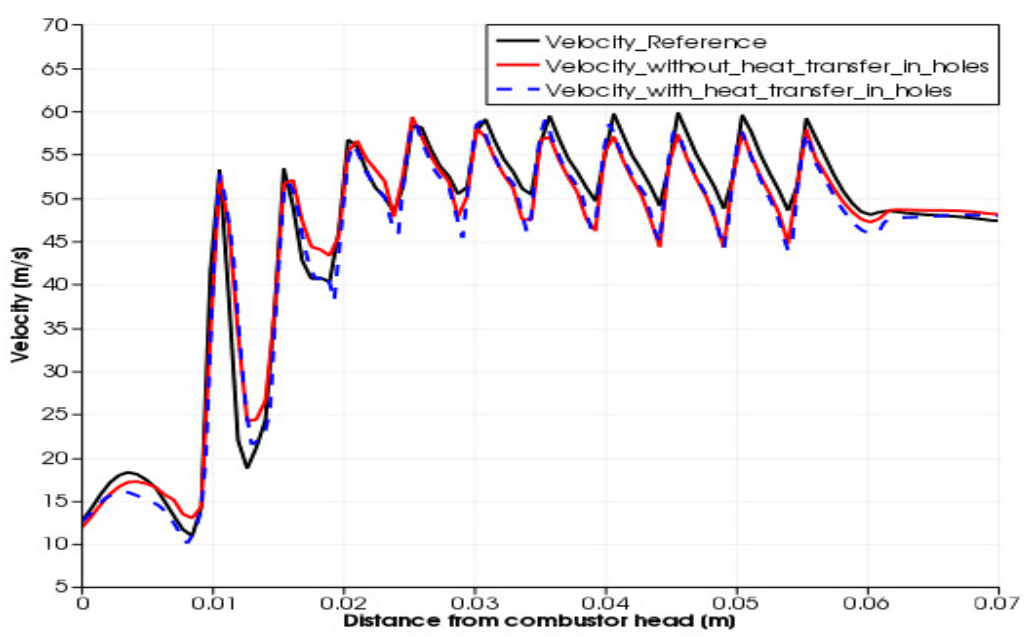

(a) Velocity at line $(\mathrm{x}=0 \mathrm{~mm}$, dist $=0.5 \mathrm{~mm}$ from the wall $)$

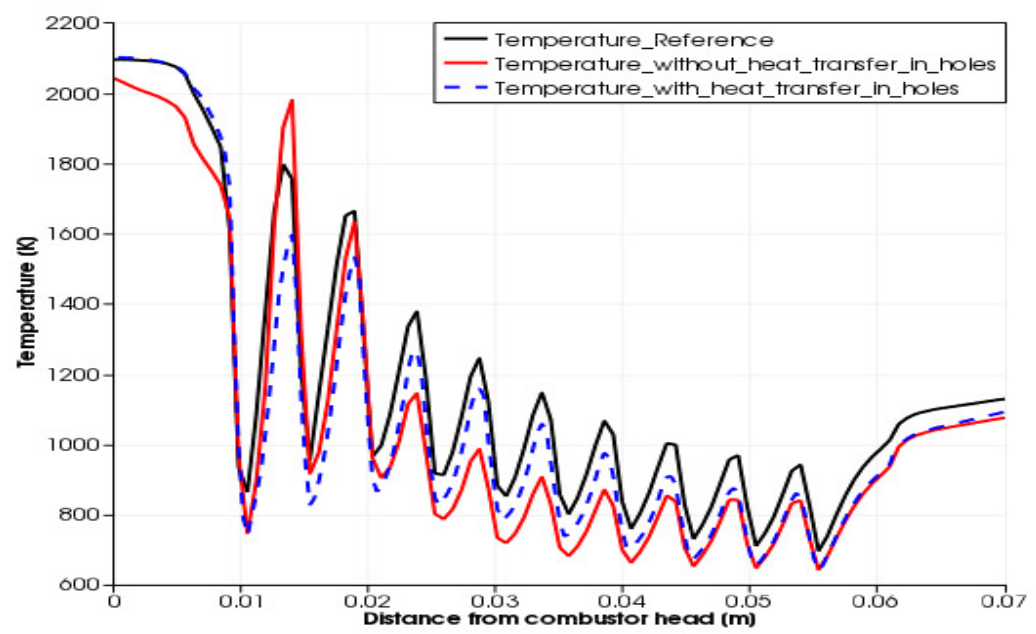

(b) Temperature at line $(\mathrm{x}=0 \mathrm{~mm}$, dist $=0.5 \mathrm{~mm}$ from the wall $)$

Figure 11: Line plot along cooled wall element with and without convective heat transfer

By using a detailed chemical reaction mechanism the study of unburned components such as $\mathrm{CO}$ is possible. The $\mathrm{CO}$ mass fraction is given in Fig. 12 at two different slices parallel to the cooled wall element. A good prediction of the $\mathrm{CO}$ formation with the modelled simulation is achieved. If the distance to the wall is increased an overestimation of the $\mathrm{CO}$ mass fraction can be seen for the modelled case. Although the results with the model are quite similar to the reference solution some differences might be observed and should be discussed. The white flagged region in Fig 12 at $0.5 \mathrm{~mm}$ from the wall, shows a long streak (a) which cannot be found (or only weak) in the modelled simulation (b). However looking at a distance of $2.5 \mathrm{~mm}$, there exists an widespread and uniformly distributed $\mathrm{CO}$ mass fraction in the model (d) compared to the reference solution (c). In the reference solution a region (see white circle in Fig. 12) with less $\mathrm{CO}$ can be found compared to the modelled simulation. Furthermore there is a higher $\mathrm{CO}$ mass fraction in downstream direction for the modelled simulation. Now the question arises, if $\mathrm{CO}$ is influenced by mixing due to the modelled cooling air distribution or by chemical reactions which prevent $\mathrm{CO}$ to be oxidized. A linear relation between mixture fraction and $\mathrm{CO}$ mass fraction exists, if $\mathrm{CO}$ changes only by mixing. At a distance of $0.5 \mathrm{~mm}$ from the wall this value is not constant whereas chemical reaction occurs. At a distance of $2.5 \mathrm{~mm}$ the differences increases. Hence in the modelled case kinetic effects are stronger. 


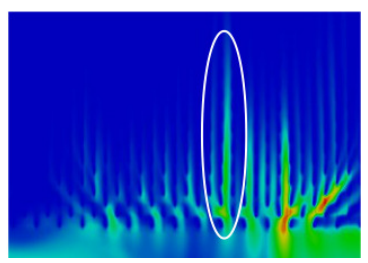

(a) Reference

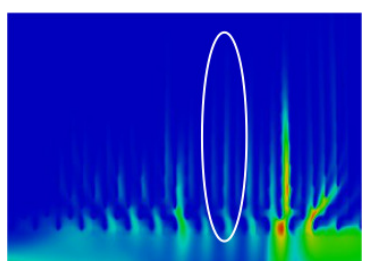

(b) Modelled

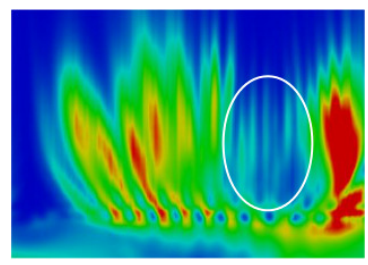

(c) Reference

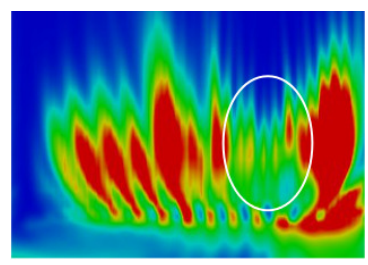

(d) Modelled

co mass fraction $(\mathrm{kg} / \mathrm{kg})$

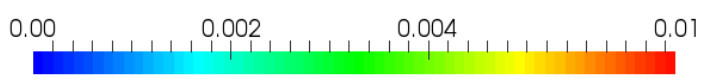

Figure 12: CO mass fraction: (a),(b) at dist $=0.5 \mathrm{~mm},(\mathrm{c}),(\mathrm{d})$ at dist $=2.5 \mathrm{~mm}$ from the wall

Activating conjugated heat transfer and heat conduction calculation in the solid under same operating conditions leads to wall temperature distribution on the hot gas side shown in Fig.13. One can observe that only small differences between reference (see Fig.13b) and modelled (see Fig.13a solution occurs. The contribution of the heat transfer inside the effusion cooling holes not only depends on the respective heat transfer coefficient but on the relative contribution of the effusion cooling holes to the overall heat removal from the wall. Hence a ratio $Z$ can be defined describing the relative contribution of the effusion cooling holes: $\alpha_{\text {eff }} \cdot A_{\text {eff }} / \sum\left(\alpha_{i} \cdot A_{i}\right)$, where the index $i$ characterizes all surfaces of heat removal. For $Z<<1$ the heat removal inside the holes has not a significant effect on the overall temperature level of the cooled wall. The parameter $\mathrm{Z}$ controls whether the heat transfer inside the cooling holes has to be taken into account in modelling effusion cooling. In cases where not only the overall temperature level is important but also the temperature homogeneity inside the wall, the parameter $\mathrm{Z}$ can be calculated with the respective Biot number $\left(B i=\alpha \cdot l / \lambda_{s}\right)$ instead of the heat transfer coefficients. Here $l$ is a characteristic length scale for each individual surface.

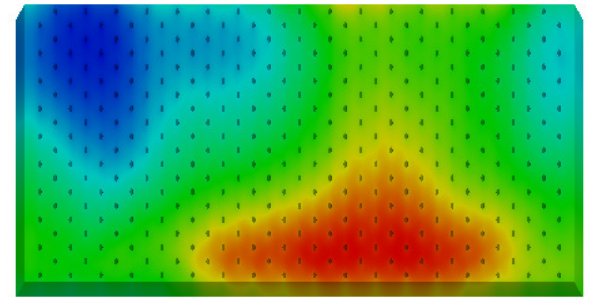

(a) Reference solution

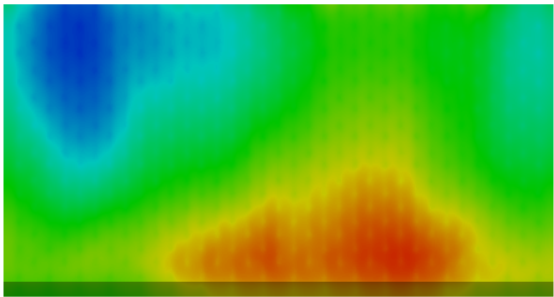

(b) Modelled - heat transfer in hole

Wall Temperature $(\mathrm{K})$

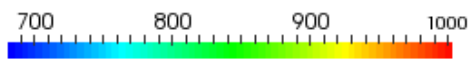

Figure 13: Wall temperature on hot gas side

\section{Summary and Conclusion}

Numerical simulations of a real combustor with an effusion cooling system have been performed. A reference simulation has been done where effusion holes are meshed and heat transfer to a cooled wall element even inside the holes was considered. To speed up calculation and to optimise the design process an effusion hole model with virtual holes has been developped which also takes convective heat transfer in each virtual hole into account. A kinetically con- 
trolled combustion model was used to predict species concentrations and its distributions near the cooled wall. It has been shown that the effusion model, even with its simple calculation of the new physical boundary conditions, is able to reproduce the solution of the real configuration. Computational time could be reduced onto on third of the real configuration for the solid and scales with the mesh size for the solid domain. The results considering wall temperatures are in a very good agreement to the reference solution. For future work a reliable tool is now available to do some more investigations concering emissions in the near wall region which has been done for the $\mathrm{CO}$ emissions. Geometrical variations for the effusion hole pattern are now able to perform.

\section{Acknowledgements}

The work was supported by the Bundesministerium für Wirtschaft und Technologie (BMWi) as per resolution of the German Federal Parliament under grant number 19K6SAJa (VALMATEC). The responsibility for the content lies solely with its authors. Furthermore the authors gratefully acknowledge their colleagues at the Institute for Propulsion Technology, for operating the test campaigns as well as for very helpful discussions. Especially we would like to thank Alexander Schroeder for his contributions from his bachelor thesis.

\section{References}

1. A. H. Lefebvre. Gas Turbine Combustion. Taylor \& Francis, ISBN 1-56032-673-5, 1999GT2012-68251, 2012.

2. S. Voigt, B. Noll, and A. Aigner. Development of a macroscopic CFD model for effusion cooling applications. ASME Turbo Expo, GT2012-68251, 2012.

3. A. Andreini, R. Da Soghe, B. Facchini, L. Mazei. Local source based CFD modelling of effusion cooling holes: Validation and Application to an actual combustor test case. Journal of Engineering for Gas Turbines and Power, Vol. 136, 011506-1, 2014.

4. S. Rida, R. Reynolds, S. Chakracorty, K. Gupta. Imprinted effusion modeling and dynamic CD calculation in gas turbine combustors. Proceedings of ASME Turbo Expo, GT201268804, 2012

5. M. Martiny. Wärmeübergang in effusionsgekühlten Flammenrohrwänden. PhD Thesis, Universität Karlsruhe, 1998

6. A. Schroeder. Implementierng von geometrischen Randbedingungen in ein bestehendes 3d CFD Effusionsbohrungsmodel. Bachalor Thesis. DLR, 2015

7. H. Hausen. Wärmeübertragung im Gegenstrom, Gleichstrom und Kreuzstrom. Springer Verlag, Berlin, 1950

8. L. Lange, J. Heinze, M. Schroll, C. Willert, T. Behrendt. Combination of Planar Laser Optical Measurement Techniques for the Investigation of Pre-mixed Lean Combustion. 16th Int. Symp. on Applications of Laser Techniques to Fluid Mechanics, Lisbon, 2012

9. Code_Saturne documentation. Practical User Guide, 2008

10. SYRTHES 3.4.1. documentation. User Manual, 2008

11. V.I. Golovitchev. Development of an Universal Model of Turbulent Spray Combustion. Chalmers University of Technology, TFR Research Proposal, Göteborg, May 2001

12. H. Schütz, R. Lückerath, B. Noll, M. Aigner. Complex Chemistry Simulation of FLOX: Flameless Oxidation Combustion. International Journal on Energy for a Clean Environment, Volume 8, Issue 3, 2007

13. A. Krazakov and M. Frenklach. GRI 1.2 Reduced Mechanism. http://www.me.berkeley.edu/drm/, 1994 